\title{
Central sleep apnea during continuous positive airway pressure therapy in obstructive sleep apnea patients: from the compliance to adaptation, maladaptation and reflexes
}

\author{
Carolina Lombardi $^{1,2}$, Sergio Caravita ${ }^{3}$, Gianfranco Parati ${ }^{1,2,3}$ \\ ${ }^{1}$ Sleep Disorders Center, San Luca Hospital, IRCCS Istituto Auxologico Italiano, Milan, Italy; ${ }^{2}$ Department of Medicine and Surgery, University \\ of Milano-Bicocca, Milan, Italy; ${ }^{3}$ Department of Cardiovascular, Neural and Metabolic Sciences, San Luca Hospital, IRCCS Istituto Auxologico \\ Italiano, Milan, Italy \\ Correspondence to: Dr. Carolina Lombardi. Head of Sleep Medicine Centre, Istituto Auxologico Italiano, Piazzale Brescia 20, Milan 20149, Italy. \\ Email: c.lombardi@auxologico.it. \\ Provenance: This is an invited Editorial commissioned by Section Editor Dr. Ning Ding (Department of Respiratory and Critical Care Medicine, The \\ First Affiliated Hospital of Nanjing Medical University, Nanjing, China). \\ Comment on: Liu D, Armitstead J, Benjafield A, et al. Trajectories of emergent central sleep apnea during continuous positive airway pressure therapy. \\ Chest 2017. [Epub ahead of print].
}

Submitted Sep 08, 2017. Accepted for publication Sep 18, 2017.

doi: $10.21037 /$ jtd.2017.09.116

View this article at: http://dx.doi.org/10.21037/jtd.2017.09.116

Obstructive sleep apnea syndrome (OSAS) is the most common form of sleep-disordered breathing. Untreated OSAS is associated with an increase in comorbidities, mortality risk, health care costs, and traffic accidents (1). However, OSAS is susceptible to treatment by various approaches, the gold standard being the application of continuous positive airway pressure (CPAP), especially when OSA is severe and when it is associated with excessive daytime sleepiness (2).

CPAP therapy has largely proven to be efficacious in reducing sleepiness, blood pressure values and cardio/ cerebrovascular risk, in particular when started in an early stage of the disease before the appearance of complications (primary prevention) and in patients who use the device for $>4$ hours per night $(3,4)$, while the benefits of its implementation in secondary prevention are more controversial (5).

Regardless its efficacy, however, a number of patients do not tolerate CPAP ventilation, either for psychological reasons or due to different methods and treatment targets in CPAP titration (6-8). Optimizing adherence to CPAP therapy in patients with obstructive sleep apnea (OSA) is thus a major challenge for sleep specialists. It has been reported that only half of patients still adhere to prescribed CPAP treatment three months after initiating therapy (9).
One particular condition recently described to be associated with poor compliance is "complex sleep apnea syndrome" (CompSAS). CompSAS is characterized by the development or persistence of central apneas or hypopneas during application of CPAP [central apnea index (CAI) $\geq 5$ events. $\left.\mathrm{h}^{-1}\right]$ in patients with predominantly obstructive apneas during the initial diagnostic sleep study. Moreover, emerging central apneas were also reported after initiation of oral appliance use, as well as after tracheostomy, maxillofacial surgery and after surgical relief of nasal obstruction (10).

The prevalence of CompSAS appears to widely vary among different studies, ranging between $56 \%$ and $18 \%$. However, the prevalence of CompSAS in a clinical setting is not so easy to define, given the dynamic features of this condition, with improvement or disappearance of this breathing pattern during sleep in some patients, and its de novo appearance in others. In general, CompSAS is more common in men, in the presence of more severe OSAS, and in patients with coronary artery disease or congestive heart failure, even if the available evidence on its occurrence is not very consistent (11-13).

Patients developing CompSAS do not show significant differences in age, level of daytime sleepiness (based on Epworth scale), lung function parameters (i.e., forced 
expiratory volume in $1 \mathrm{~s}$ ) or arterial blood gases as compared with patients not developing CompSAS. Moreover, based on the diagnostic sleep study, it is not possible to predict whether someone will develop CompSAS $(14,15)$.

Some studies have shown that in the majority of patients with CompSAS, CAI lowers significantly during followup, with improvement in sleep efficiency, a decrease in wake after sleep onset, and a lower number of arousals in comparison with the baseline diagnostic night (16). However, CPAP seems to be effective in some patients, while ineffective in others; CompSAS may develop de novo in $4 \%$ of OSAS patients treated with CPAP during follow up (17). Very few data are available on CPAP compliance, but there are suggestions that CompSAS patients may have a lower effect of CPAP on sleep as well as on breathing parameters, more dyspnea, poorer CPAP adherence and more often report spontaneous removal of the mask at night (18).

A recent work by Liu et al. (19) explored trajectories of treatment-emergent central sleep apnea (CSA) during CPAP therapy, obtained through the analysis of a CPAP database of 133,006 patients from the U.S. telemonitoring device data-AirView, who used CPAP for $\geq 90$ days and had $\geq 1$ day with use of $\geq 1 \mathrm{~h}$ in week 1 and week 13 . After the analysis of the presence/absence of emergent CSA at baseline (week 1) and week 13, four groups were defined as follows: OSA (average CAI $<5 / \mathrm{h}$ in week $1,<5 / \mathrm{h}$ in week 13); transient CSA (CAI $\geq 5 / \mathrm{h}$ in week $1,<5 / \mathrm{h}$ in week 13 ); persistent CSA (CAI $\geq 5 / \mathrm{h}$ in week $1, \geq 5 / \mathrm{h}$ in week 13 ); emergent CSA (CAI $<5 / \mathrm{h}$ in week $1, \geq 5 / \mathrm{h}$ in week 13 ). Patients with CSA during CPAP therapy were older, had higher residual AHI and CAI; patients with emergent CSA also had significantly higher air leak during CPAP in the first 90 days.

Compliance to CPAP, reflected by average number of daily usage hours in the first 90 days, was lower in those who did vs. those who did not develop CSA during CPAP therapy: mean average use was $5.97 \mathrm{~h} / \mathrm{d}(95 \% \mathrm{CI}, 5.96-5.98)$ in those without CSA, $5.75 \mathrm{~h} / \mathrm{d}(5.68-5.83)$ in those with transient CSA, $5.87 \mathrm{~h} / \mathrm{d}(5.75-5.99)$ in those with persistent CSA, and $5.66 \mathrm{~h} / \mathrm{d}(5.52-5.80)$ in patients with emergent CSA. Additionally, patients with any CSA during CPAP were significantly more likely to terminate therapy after 90 days compared with those who did not develop CSA.

The estimated probability of continuing CPAP therapy on day 300 was $83 \%$ for the OSA group, and $79 \%, 76 \%$ and $72 \%$ for the transient CSA, persistent CSA and emergent CSA groups, respectively. The authors are to be commended for these results that included a large sample of patients from real-life, further providing 1-week assessment windows and repeated measures based on telemonitoring data. Nonetheless, some limitations of this study need to be acknowledged. In particular, information on baseline AHI and type of sleep study conducted for titration (full night titration $v s$. split night), anthropometric parameters, comorbidities or drug treatment changes that may have influenced the reported prevalence rates of CSA, is not reported. Moreover, the population was heterogeneous in terms of treatment, including both patients using Auto CPAP and fixed CPAP; and the ventilatory parameters during sleep have been obtained from a ventilatory support device rather than from a polysomnographic study.

But how to deal with these results? How to interpret them in the perspective of more efforts needed to increase compliance and efficacy of CPAP therapy? Liu et al. conducted an epidemiological work that raises questions more than providing answers. Some explanation for their results might only be inferred from pathophysiological reasoning, an issue, however, that might need to be specifically addressed in future studies. Indeed, the pathogenesis of CompSAS is not fully clarified and is likely related to a complex combination of reflex mechanisms (Figure 1). In particular, it might be hypothesized that (mal)adaptive responses to chronic intermittent upper airway obstruction during sleep (including, but not limited to, the development of diastolic heart failure, fluid overload and chemoreflex desensitization) may predispose to ventilatory control instability (20). However, such instability of ventilatory control may not become apparent until correction of obstructive events by CPAP, since upper airway obstruction may represent a stronger stimulus to maintain ventilation, "paradoxically" overriding instability in these control mechanisms.

A similar scenario has been recently described for ventilatory control during exercise in patients with postcapillary pulmonary hypertension due to left heart disease, a condition normally characterized by a high prevalence of exercise oscillatory breathing. In this context, the superposition of a pre-capillary component to pulmonary hypertension (as defined by a combination of increased pulmonary vascular resistance and pulmonary vascular pressure gradients) is associated with right heart chamber stretch and steeper ventilatory responses to exercise. The latter seems to override ventilatory control instability, being thus associated with less than expected exercise oscillatory breathing (21). 


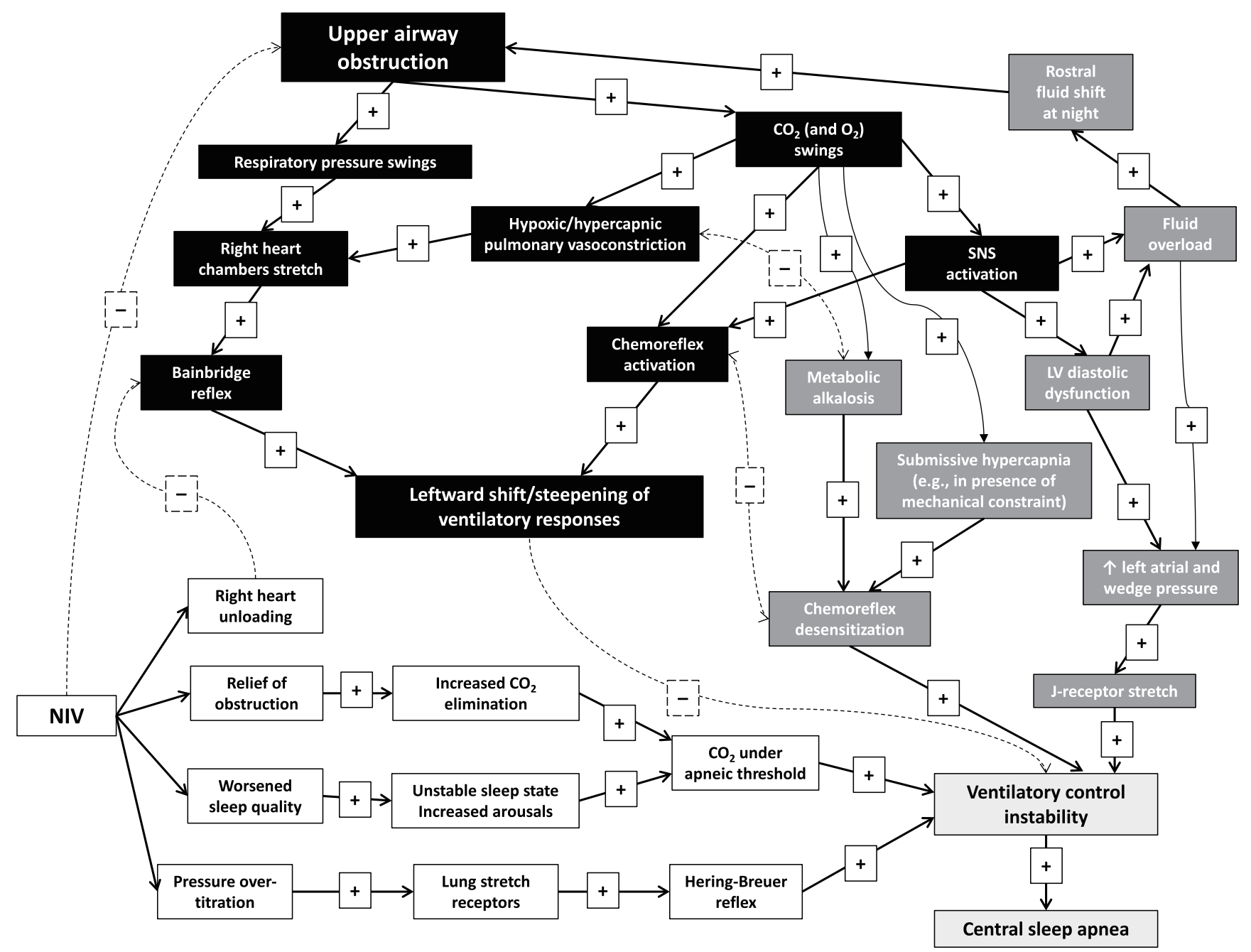

Figure 1 Presumptive pathophysiological mechanisms underlying complex sleep apneas. Black boxes: cascade of events secondary to obstructive sleep apneas, intervening soon in the course of this disease and favoring chemoreflex activation with leftward shift and steepening of the ventilatory responses to chemical stimuli. Dark gray boxes: cascade of events secondary to obstructive sleep apneas, intervening later in the course of the disease (maladaptive), eventually favored by comorbidities (e.g., heart failure with fluid retention and increase in cardiac filling pressures; obesity or obstructive lung disorders with mechanical constraint) and promoting ventilatory control instability and central sleep apnea (light gray boxes). White boxes: cascade of events secondary to initiation of nocturnal ventilation, eventually promoting ventilatory control instability. Solid lines indicate positive interactions (+); dashed lines indicate inhibitory (-) interactions. $\mathrm{CO}_{2}$, carbon dioxide; $\mathrm{O}_{2}$, oxygen; LV, left ventricle; NIV, non-invasive ventilation; SNS, sympathetic nervous system.

More in detail, several reflex mechanisms might stepin to explain ventilatory control instability leading to CompSAS, when airways obstruction is relieved by specific OSA treatment, depending on the characteristics of the individual patient, namely:

* Increased carbon dioxide $\left(\mathrm{CO}_{2}\right)$ excretion during CPAP application (or other OSA treatment), reducing arterial carbon dioxide partial pressure
$\left(\mathrm{PaCO}_{2}\right)$ below the apneic threshold. It should be also kept in mind that the apneic threshold might be rightward shifted in the setting of longstanding OSA either by the development of metabolic alkalosis compensating for $\mathrm{CO}_{2}$ retention during sleep time, or in the presence of concomitant mechanical or physiological constraint leading to submissive hypercapnia (e.g., in the setting of comorbidities 
such as obesity or obstructive lung disorders) (20).

* The presence of an underlying left ventricular diastolic dysfunction (heart failure with preserved ejection fraction), that may promote per se CSA as a result of increased left atrial pressure and relative volume overload $(20,22)$, and whose diagnosis may not be straightforward, especially in its earliest phases (23).

* Right heart unloading by CPAP with reduced stretch of mechanoreceptors (21) which would otherwise promote hyperventilation overriding ventilatory control instability.

* Over-titration of CPAP with activation of stretch receptors in the lungs. When these receptors are activated due to an excessive expansion of the lungs, they send signals to the breathing center along the vagal nerve fibers, which inhibit the central motor output. Hence, this will result in an interruption of inspiration. This mechanism protects the lungs against overexpansion, and is called the HeringBreuer reflex (16). Moreover, in some patients (e.g., those more preload dependent or those with low ventricular filling pressures) CPAP over-titration may determine a drop of cardiac output, which may further promote ventilatory control instability due to circle time lengthening.

* worsening of sleep quality by CPAP in some patients. The frequent changes from sleep to wake and from wake to sleep lead to instability in the ventilatory control system. This leads to oscillations in the $\mathrm{PaCO}_{2}$ values that can lead to a decrease in $\mathrm{PaCO}_{2}$ below the apneic threshold, with inherent central apneas as a consequence. It remains unclear to what extent sleep impairment is the cause or the effect of CompSAS (24). It was suggested that an elevated nasal resistance might relate to frequent arousals, which could presumably contribute to an increase in central apneas (25).

* All these pathophysiological issues may be clinically important, since they can potentially affect treatment approaches, keeping in mind that patients developing CSA after OSAS treatment seem to have a poorer prognosis as compared to those only affected by OSAS, eventually also because of low CPAP compliance.

While we still cannot answer the question whether CSA after the onset of CPAP is a new pattern rather than a preexisting masked phenomenon, we may hypothesize that both these possibilities can hold true, depending on the individual patient's characteristics. Only a more accurate patients' phenotyping is likely to provide us with clearer answers in this controversial setting, helping us to identify the more adequate treatment for a given patient.

\section{Acknowledgements}

None.

\section{Footnote}

Conflicts of Interest: The authors have no conflicts of interest to declare.

\section{References}

1. Parati G, Lombardi C, Narkiewicz K. Sleep apnea: epidemiology, pathophysiology, and relation to cardiovascular risk. Am J Physiol Regul Integr Comp Physiol 2007;293:R1671-83.

2. Cao MT, Sternbach JM, Guilleminault C. Continuous positive airway pressure therapy in obstuctive sleep apnea: benefits and alternatives. Expert Rev Respir Med 2017;11:259-72.

3. Jennum P, Tønnesen P, Ibsen R, et al. Obstructive sleep apnea: effect of comorbidities and positive airway pressure on all-cause mortality. Sleep Med 2017;36:62-6.

4. Abuzaid AS, Al Ashry HS, Elbadawi A, et al. Meta-analysis of cardiovascular outcomes with continuous positive airway pressure therapy in patients with obstructive sleep apnea. Am J Cardiol 2017;120:693-9.

5. McEvoy RD, Antic NA, Heeley E, et al. CPAP for prevention of cardiovascular events in obstructive sleep apnea. N Engl J Med 2016;375:919-31.

6. Maschauer EL, Fairley DM, Riha RL. Does personality play a role in continuous positive airway pressure compliance? Breathe (Sheff) 2017;13:32-43.

7. Nadal N, de Batlle J, Barbé F, et al. Predictors of CPAP compliance in different clinical settings: primary care versus sleep unit. Sleep Breath 2017. [Epub ahead of print].

8. Berry RB, Parish JM, Hartse KM. The use of autotitrating continuous positive airway pressure for treatment of adult obstructive sleep apnea. An American Academy of Sleep Medicine review. Sleep 2002;25:148-73.

9. McMillan A, Bratton DJ, Faria R, et al. A multicentre randomised controlled trial and economic evaluation of 
continuous positive airway pressure for the treatment of obstructive sleep apnoea syndrome in older people: PREDICT. Health Technol Assess 2015;19:1-188.

10. Randerath W, Verbraecken J, Andreas S, et al. Definition, discrimination, diagnosis and treatment of central breathing disturbances during sleep. Eur Respir J 2017;49. pii:1600959.

11. Marrone O, Stallone A, Salvaggio A, et al. Occurrence of breathing disorders during CPAP administration in obstructive sleep apnea syndrome. Eur Respir J 1991;4:660-6.

12. Bitter T, Westerheide N, Hossain MS, et al. Complex sleep apnoea in congestive heart failure. Thorax 2011;66:402-7.

13. Westhoff $M$, Arzt M, Litterst P. Prevalence and treatment of central sleep apnoea emerging after initiation of continuous positive airway pressure in patients with obstructive sleep apnoea without evidence of heart failure. Sleep Breath 2012;16:71-8.

14. Morgenthaler TI, Kagramanov V, Hanak V, et al. Complex sleep apnea syndrome: is it a unique clinical syndrome? Sleep 2006;29:1203-9.

15. Mulgrew AT, Lawati NA, Ayas NT, et al. Residual sleep apnea on polysomnography after 3 months of CPAP therapy: clinical implications, predictors and patterns. Sleep Med 2010;11:119-25.

16. Dernaika T, Tawk M, Nazir S, et al. The significance and outcome of continuous positive airway pressure related central sleep apnea during split-night sleep studies. Chest
2007;132:81-7.

17. Cassel W, Canisius S, Becker HF, et al. A prospective polysomnographic study on the evolution of complex sleep apnoea. Eur Respir J 2011;38:329-37.

18. Verbraecken J. Complex sleep apnoea syndrome. Breathe 2013;9:373-80.

19. Liu D, Armitstead J, Benjafield A, et al. Trajectories of emergent central sleep apnea during CPAP therapy. Chest 2017. [Epub ahead of print].

20. Caravita S, Vachiéry JL. Obstructive ventilatory disorder in heart failure-caused by the heart or the lung? Curr Heart Fail Rep 2016;13:310-8.

21. Caravita S, Faini A, Deboeck G, et al. Pulmonary hypertension and ventilation during exercise: Role of the pre-capillary component. J Heart Lung Transplant 2017;36:754-62.

22. Parati G, Lombardi C, Castagna F, et al. Heart failure and sleep disorders. Nat Rev Cardiol 2016;13:389-403.

23. Borlaug BA, Nishimura RA, Sorajja P, et al. Exercise hemodynamics enhance diagnosis of early heart failure with preserved ejection fraction. Circ Heart Fail 2010;3:588-95.

24. Malhotra A, Bertisch S, Wellman A. Complex sleep apnea: it isn't really a disease. J Clin Sleep Med 2008;4:406-8.

25. Salloum A, Rowley JA, Mateika JH, et al. Increased propensity for central apnea in patients with obstructive sleep apnea: Effect of nasal continuous positive airway pressure. Am J Respir Crit Care Med 2010;181:189-93.
Cite this article as: Lombardi C, Caravita S, Parati G. Central sleep apnea during continuous positive airway pressure therapy in obstructive sleep apnea patients: from the compliance to adaptation, maladaptation and reflexes. J Thorac Dis 2017;9(11):4152-4156. doi: 10.21037/jtd.2017.09.116 